\title{
Migration of Cymetra After Vocal Fold Injection for Laryngeal Paralysis
}

Jonathan M. Bock, MD; John H. Lee, MD; Robert A. Robinson, MD, PhD; Henry T. Hoffman, MD

Injection laryngoplasty is a common operative technique used in the management of unilateral vocal fold paralysis. Cymetra is a micronized particulate injectable form of acellular human dermis that is commonly used for vocal cord medialization procedures. We report migration of an intracordal bolus of Cymetra into the medial wall of the pyriform sinus. Histopathology from endoscopic resection of the migrated Cymetra demonstrated a localized foreign body reaction with characteristic giant cells. Specific anatomic considerations through cadaveric laryngeal dissection are presented to demonstrate the likely pathway of intralaryngeal bolus migration.

Key Words: Vocal cord paralysis, Cymetra, micronized AlloDerm, injection laryngoplasty, paraglottic space, thyroarytenoid gap, complications.

Laryngoscope, 117:2251-2254, 2007

\section{INTRODUCTION}

Initial attempts to treat vocal cord paralysis with injection laryngoplasty began early in the 1900s with paraffin injections. Subsequent reports of injections using both autologous and synthetic injectable substances over the past century have revealed both beneficial and morbid outcomes. Teflon (polytetrafluoroethylene [PTFE]) was introduced to the American public as a commercial product by the Dupont corporation in 1946 and was first published for use in injection laryngoplasty in 1962 by Lewy. The use of Teflon has decreased markedly after the identification of long-term complications such as foreign body reactions, granuloma formation, and lymphatic migration of PTFE particles.

Bovine collagen injection for vocal fold augmentation was first published by Ford in 1984. Initial studies supported the utility of bovine collagen for intracordal injec-

From the Departments of Otolaryngology—Head and Neck Surgery (J.M.B., J.H.L., H.T.H.) and Pathology (R.A.R.), University of Iowa Health Care, Iowa City, Iowa, U.S.A.

Editor's Note: This Manuscript was accepted for publication June 21, 2007.

Send correspondence to Dr. Henry T. Hoffman, Department of Otolaryngology-Head and Neck Surgery, University of Iowa Hospitals and Clinics, 200 Hawkins Drive, Iowa City, IA 52242. E-mail: henryhoffman@uiowa.edu

DOI: 10.1097/MLG.0b013e3181462a16 tion because of decreased immunologic foreign body reactions compared with Teflon. Injection laryngoplasty with collagen has become popular because of the relative ease of injection, an established safety record from previous use in dermatologic injections, and the natural endogenous presence of collagen within the lamina propria of the vocal fold. Concerns about hypersensitivity reactions to bovine antigens in $3 \%$ of patients led to the development of both autologous and cadaveric collagen injection techniques in the $1990 \mathrm{s.}^{1}$

AlloDerm (Life Cell Corporation, Branchburg, NJ) is an acellular dermal matrix composed of collagen, hyaluron, fibronectin, and other extracellular matrix components that is derived from donated human skin tissue. AlloDerm was introduced as an alternative grafting material for burn patients in $1995,{ }^{2}$ and subsequent studies demonstrated that it favorably promoted host collagen deposition and fibroblast ingrowth in addition to decreased host immune response compared with other contemporary injection modalities. Cymetra is a micronized particulate injectable form of AlloDerm that saw widespread clinical use for facial rhytid injections beginning in 2002. Subsequent studies demonstrated the efficacy of Cymetra for injection laryngoplasty, ${ }^{3}$ and its use has become widespread among both laryngologists and general otolaryngologists.

Currently, multiple different products are available for injection laryngoplasty, including collagen, fat, carboxymethylcellulose, hyaluronic acid, and calcium hydroxylapatite. Despite improvements in surgical instrumentation and technique, complications such as airway compromise, implant migration, and suboptimal voice outcomes result in a revision surgery rate for injection laryngoplasty when using these materials that exceeds $5 \% .4$

To date, few complications have been published regarding Cymetra for injection laryngoplasty. Reported complications have been limited to the development of an intralaryngeal abscess after Cymetra injection ${ }^{5}$ and the formation of firm submucosal masses within Reinke's space that interfered with mucosal wave propagation after superficial injection. ${ }^{6}$ We discuss a case of migration of Cymetra from the vocal cord to the medial wall of the pyriform sinus requiring surgical excision to address subsequent symptoms of odynophagia and dysphagia. Histopathology of the excised Cymetra showing a foreign body 
reaction is discussed. Cadaveric laryngeal dissections are used to demonstrate the likely pathway of migration of the Cymetra bolus.

\section{CASE REPORT}

An 18-year-old female college student presented to the University of Iowa Otolaryngology clinic for evaluation of breathy dysphonia that had been present for many years. Vocal music studies in college exacerbated this dysphonia and prompted her evaluation. As an infant, vocal cord paralysis caused by birth trauma had been identified and had presumably resolved without subsequent intervention. She had not been evaluated by an otolaryngologist for this problem since she was a child. Clinical evaluation demonstrated an immobile left vocal cord associated with incomplete glottic closure, slight supraglottic compression, and a mild degree of dysphonia characterized by breathiness. Magnetic resonance imaging (MRI) of the brain to the chest was ordered to rule out a Chiari malformation or mass and was negative for any abnormalities.

The patient received two injections of Cymetra into the left true vocal cord (TVC) over 4 months time to augment her left vocal fold, one via an external approach in the clinic and a second injection in the operating room via standard direct operative laryngoscopy. A total of 1.5 $\mathrm{mL}$ of Cymetra was injected during the first injection followed by $0.75 \mathrm{~mL}$ during the second injection.

She returned to the clinic 4 weeks after her second injection with continued complaints of vocal asthenia, breathiness, and decreased pitch range, which correlated with the findings of a large posterior glottic chink and decreased bulk of the immobile left TVC. The patient requested further injections to improve her voice, and therefore a third injection of an additional $1.2 \mathrm{~mL}$ of Cymetra was injected into the left mid-cord by standard direct laryngoscopy technique. Endoscopic photographs were obtained at the completion of the injection showing the medialized vocal cord (Fig. 1) with the cuff of the endotracheal tube in a very high position in the subglottis.

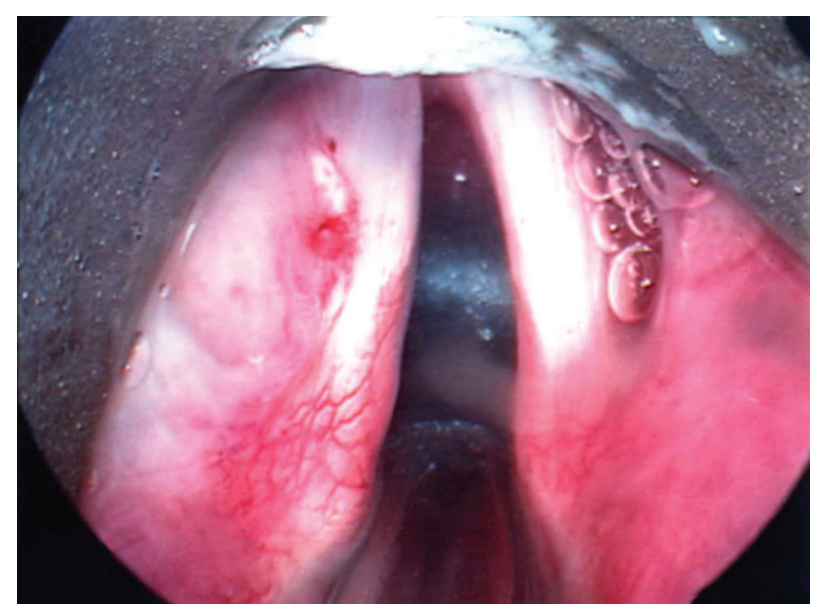

Fig. 1. Intraoperative endoscopic view immediately postinjection of Cymetra. Note the high subglottic position of the endotracheal tube cuff.

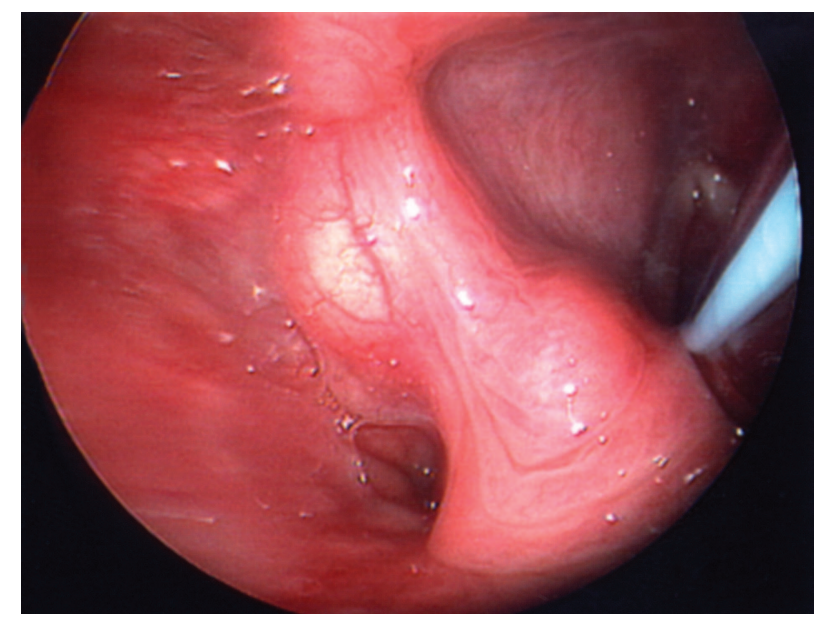

Fig. 2. Intraoperative endoscopic view of the left pyriform sinus showing Cymetra mass within the aryepiglottic fold.

The patient recovered from this third injection without immediate complications and was discharged home.

The patient returned to the voice clinic 5 weeks after her third injection with continued complaints of a weak voice as well as progressive dysphagia and odynophagia without fevers or chills. Physical examination showed decreased bulk of the left TVC and a submucosal mass within the lateral aspect of the left aryepiglottic (AE) fold pushing into the pyriform sinus. Because of progressively worsening odynophagia and dysphagia, microdirect laryngoscopy was performed for endoscopic removal of the left AE fold mass (Fig. 2). Under microscopic guidance, an incision was made through the mucosa along the longitudinal axis parallel to the $\mathrm{AE}$ fold. Careful blunt dissection revealed a $1.1 \times 0.6 \times 0.3 \mathrm{~cm}$ encapsulated mass adherent to the deep tissue of the pyriform sinus. The submucosal superior laryngeal nerve was identified in the lateral aspect of the dissection and preserved. The pyriform sinus mucosal incision was closed endoscopically with 6-0 Vicryl suture. Specimens were sent for histopathology as well as culture. Histology showed an acellular collagenous material surrounded by fibrosis and a localized giant cell foreign body reaction with chronic inflammation, representing the migrated Cymetra (Fig. 3). Cultures showed small amounts of mixed oral flora without yeast or fungal elements. The patient identified significant improvement in her odynophagia immediately after surgery and was discharged home after overnight observation.

At follow-up 1 week later, her pharyngeal incision had healed well, and she had no odynophagia. The patient subsequently underwent a formal left-sided medialization thyroplasty with Gore-Tex 5 weeks after excision of the Cymetra and now has much improved voice both for singing and speaking and continues her music studies at the University of Iowa.

\section{Antomic Dissection}

A fresh female cadaveric larynx was obtained from a cadaver donated to the University of Iowa Deeded Body program. This larynx was dissected to evaluate the likely 
Fig. 3. Anatomic histopathology of explanted Cymetra bolus (hematoxylin-eosin). (A) Normal collagen present within the middle of the Cymetra mass, with pink fibrillar material present. (B) Same collagen in the superior aspect of the slide, with the formation of a pseudocapsule beneath. This capsule contains some native collagen within it as well as an occasional giant cell, indicating a foreign body reaction within the larynx.
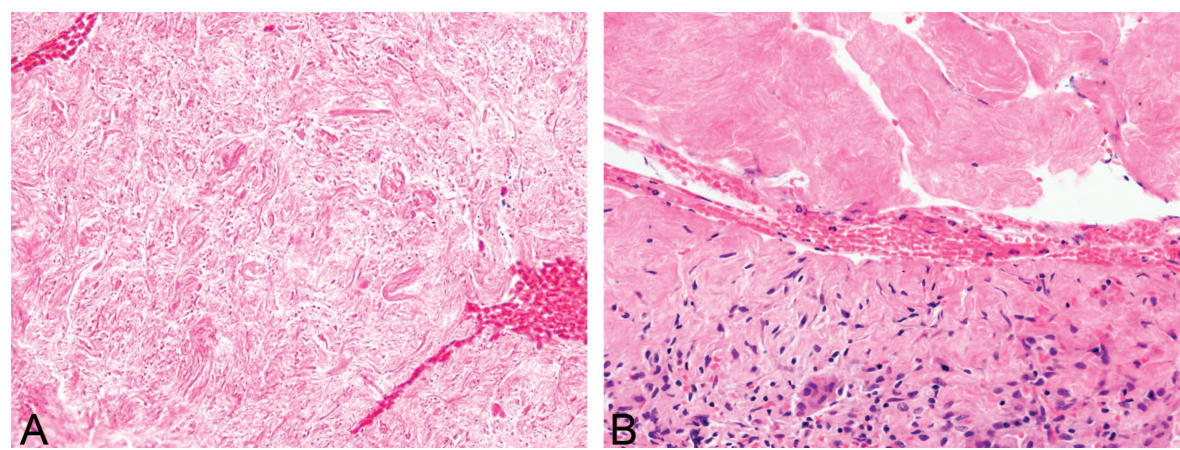

path of migration of the Cymetra bolus from the left TVC into the lateral left $\mathrm{AE}$ fold and medial wall of the pyriform sinus (Fig. 4). An incision was made over the posterior aspect of the false vocal fold near the insertion into the arytenoid, and the mucosa and muscle of the false cord were retracted anteriorly. Blunt dissection easily facilitated opening of the paraglottic space, a potential space bordered medially by the ventricle and laterally by the inner surface of the thyroid cartilage. Immediately lateral and superior to the paraglottic space, near the transition of the $\mathrm{AE}$ fold into the ligamentous support for the false vocal fold, a small muscle was noted within the $\mathrm{AE}$ fold that has previously been identified as the palatolaryngeus muscle or the aryepiglotticus muscle. This muscle band is often contiguous with the palatopharyngeus muscle ${ }^{7}$ and likely functions in deglutition. With muscular contraction of the false cord or upward pressure from an endotracheal tube, this muscle fiber band could direct any bolus of collagen up from the paraglottic space into the thyroarytenoid gap and into the lateral wall of the $\mathrm{AE}$ fold, similar to the pathway seen in this case report.

\section{DISCUSSION}

Complications from injection laryngoplasty are not a new phenomenon. Indeed, reports of Teflon granuloma formation and immune reactions arose just years after it was first used for vocal fold medialization. ${ }^{8}$ Migration of biomaterials is an acknowledged risk of injection laryngoplasty. ${ }^{4}$

To our knowledge, this case is the first to represent this specific path of migration. One previous report described an anterior migration of Teflon into the precricoid space, likely via Broyle's ligament, mimicking a cartilaginous mass. ${ }^{9}$ In our case, serial clinical examinations showed progressive movement of the bolus of collagen into the pyriform sinus with worsening progressive odynophagia and dysphagia, warranting endoscopic excision of the collagen mass. We propose that caudal force applied from a high-riding subglottic endotracheal tube cuff may have lead to upward force on the collagen bolus, dislodging the collagen bolus from its proper location. As time progressed, through continued speech and swallowing, muscular contractions of the larynx continued to push the Cymetra bolus superiorly and laterally, eventually diverting the bolus into the lateral $\mathrm{AE}$ fold. The relatively loose areolar tissue of the paraglottic space would facilitate bolus migration in this fashion, as was confirmed by our cadaveric dissections. This patient's evident supraglottic contractions with speech in voice laboratory testing also could increase the migration force applied to the Cymetra within her larynx. Correct placement of the bolus of Cymetra into the deeper layers of the lamina propria is critical to maintain mucosal wave formation and may also prevent subsequent migration of the collagen bolus into extralaryngeal locations. It is also possible that coughing or "bucking" on the endotracheal tube during anesthetic emergence could have lead to mobilization of the Cymetra bolus in this patient. Deep extubation with mask ventilation or emergence using a laryngeal mask airway may conceivably reduce stress forces on the collagen bolus and help to maintain the bolus within the true cord and prevent implant migration.

Cymetra has quickly progressed to widespread clinical use within the last 5 years largely because of its ease
Fig. 4. Cadaveric laryngeal dissections of the paraglottic space and thyroarytenoid gap demonstrating the likely path of collagen migration into the aryepiglottic fold and pyriform sinus. (A) Anterior view with arrows delineating likely path of Cymetra migration. (B) Posterior view.

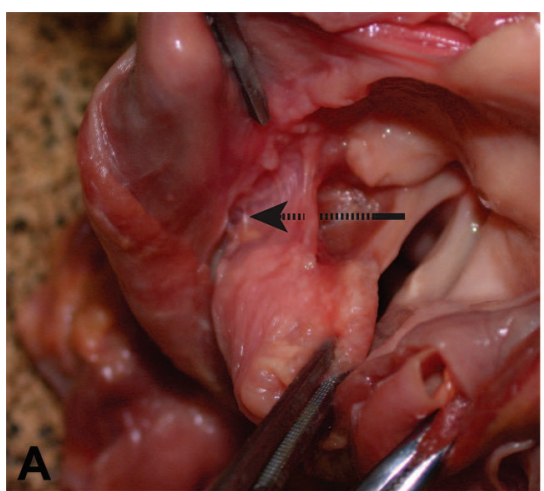

Anterior View

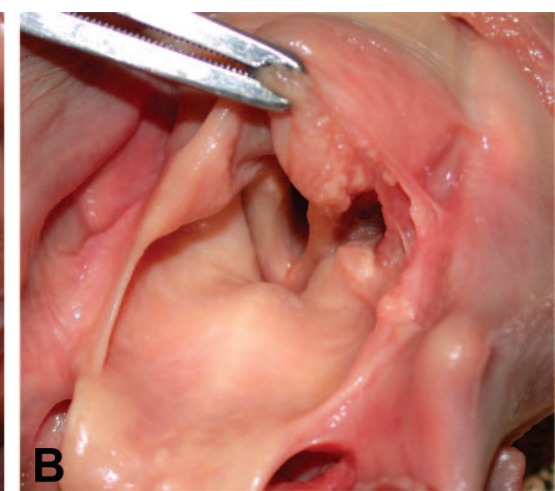

Posterior View 
of use, its resorbable nature (6-12 mo) that allows for its use as a temporizing measure, and its relatively low risk of host immune response. ${ }^{10}$ Previous experience with bovine collagen products indicated that a foreign body reaction could develop, decreasing enthusiasm for its routine clinical use. To our knowledge, no previous study has published the histopathology of explanted Cymetra after injection into a human larynx. Our data show evidence of a localized immune response to Cymetra, including a foreign body reaction characterized by the presence of giant cells along with native collagen deposition and formation of a pseudocapsule around the Cymetra bolus. Previous studies using AlloDerm, the parent compound to Cymetra, have demonstrated mixed results regarding antigraft host immune responses. A study of AlloDerm injected into the quadriceps muscle of rats showed the onset of a foreign body reaction 6 months after injection, although the local inflammatory response was judged as mild. ${ }^{11}$ One study addressed host immune response to AlloDerm implanted into human skin in a prospective trial and showed no significant local tissue reaction after 3 months of implantation in human skin. ${ }^{12}$

We identified a foreign body reaction to Cymetra on pathologic examination of the explanted operative resection specimen. It is conceivable that this immune response would not have occurred had a smaller volume of Cymetra been delivered. Three separate injections to the left vocal cord occurred within a 6-month window and delivered a total volume approximately $3.5 \mathrm{~mL}$. It is also not certain which of the three injection boluses of Cymetra was responsible for this migration and foreign body reaction. A recent study on Cymetra viability using MRI of patients postinjection showed persistence within the thyroarytenoid muscle of up to 11 months. ${ }^{13}$ Vocal improvement has been reported up to a year or more after a single vocal fold injection with Cymetra, suggesting prolonged graft life. ${ }^{14}$ Additional evidence exists for the increasing persistence of Cymetra with repeated injections,. ${ }^{15}$ This prolonged graft presence within the larynx may be responsible for the observed foreign body reaction seen in our patient. This histopathologic finding argues for further studies on the local host tissue immune response to Cymetra in the larynx.

\section{CONCLUSIONS}

Cymetra has a low complication rate when used for injection laryngoplasty in the treatment of glottic incompetence. We report successful revision surgery to address symptoms from Cymetra displaced from the vocal cord to the pyriform sinus. We also describe a localized foreign body reaction to the injected Cymetra. Further study is indicated to identify in vivo host immune responses to Cymetra and other biomaterials used for injection laryngoplasty.

\section{Acknowledgments}

The authors thank Kay Klein for assistance with manuscript preparation and the personnel in the University of Iowa Deeded Body Program for assistance with cadaveric dissections.

\section{BIBLIOGRAPHY}

1. Ford C, Staskowski P, Bless D. Autologous collagen vocal fold injection: a preliminary clinical study. Laryngoscope 1995; 105:944-948.

2. Wainwright D. Use of an acellular allograft dermal matrix (AlloDerm) in the management of full-thickness burns. Burns 1995;21:243-248.

3. Karpenko A, Dworkin J, Meleca R, Stachler R. Cymetra injection for unilateral vocal fold paralysis. Ann Otol Rhinol Laryngol 2003;112:927-934.

4. Rosen C. Complications of phonosurgery: results of a national survey. Laryngoscope 1998;108:1697-1703.

5. Zapanta PE, Steven A, MD. Laryngeal Abscess after Injection Laryngoplasty with Micronized AlloDerm. Laryngoscope 2004;114:1522-1524.

6. Anderson T, Sataloff R. Complications of collagen injection of the vocal fold: report of several unusual cases and review of the literature. $J$ Voice 2004;18:392-397.

7. Van Daele D, Perlman A, Cassell M. Intrinsic fibre architecture and attachments of the human epiglottis and their contributions to the mechanism of deglutition. $J$ Anat 1995;186:1-15.

8. Rubin H. Misadventures with injectable polytef(Teflon). Arch Otolaryngol 1975;101:114-116.

9. Jol J, Seedat R, Skinner D. A precricoid swelling in a patient treated with Teflon injection in the vocal fold after idiopathic left vocal fold palsy. J Laryngol Otol 1998;112: 878-879.

10. Pearl A, Woo P, Ostrowski R, Mojica J, Mandell D, Costantino P. A preliminary report on micronized AlloDerm injection laryngoplasty. Laryngoscope 2002;112:990-996.

11. Sengor A, Aydin O, Mola F, Gurbuz Y. Evaluation of alloderm and autologous skin in quadriceps muscles of rats for injection laryngoplasty. Eur Arch Otorhinolaryngol 2005; 262:107-112.

12. Sclafani A, Romo TR, Jacono A, McCormick S, Cocker R, Parker A. Evaluation of acellular dermal graft in sheet (AlloDerm) and injectable (micronized AlloDerm) forms for soft tissue augmentation. Clinical observations and histological analysis. Arch Facial Plast Surg 2000;2:130-136.

13. Moonis G, Dyce O, Loevner L, Mirza N. Magnetic resonance imaging of micronized dermal graft in the larynx. Ann Otol Rhinol Laryngol 2005;114:593-598.

14. Milstein CF, Akst LM, Hicks MD, Abelson TI, Strome M. Long-term effects of micronized alloderm injection for unilateral vocal fold paralysis. Laryngoscope 2005;115: 1691-1696.

15. Sclafani AP, Romo Iii T, Jacono AA. Rejuvenation of the aging lip with an injectable acellular dermal graft (Cymetra). Arch Facial Plast Surg 2002;4:252-257. 